\title{
Optimized interval 2-tuple linguistic aggregation operator based on PGSA and its application in MAGDM
}

\author{
ZONG Mengting ${ }^{1}$, SHEN Tian ${ }^{2, *}$, and CHEN Xi \\ 1. School of Business, Nanjing University, Nanjing 210000, China; \\ 2. School of Education, Nanjing University of Traditional Chinese Medicine, Nanjing 210000, China
}

\begin{abstract}
This study proposes a multiple attribute group decisionmaking (MAGDM) approach on the basis of the plant growth simulation algorithm (PGSA) and interval 2-tuple weighted average operators for uncertain linguistic weighted aggregation (ULWA). We provide an example for illustration and verification and compare several aggregation operators to indicate the optimality of the assembly method. In addition, we present two comparisons to demonstrate the practicality and effectiveness of the proposed method. The method can be used not only to aggregate MAGDM problems but also to solve multi-granularity uncertain linguistic information. Its high reliability, easy programming, and high-speed calculation can improve the efficiency of ULWA characteristics. Finally, the proposed method has the exact characteristics for linguistic information processing and can effectively avoid information distortion and loss.
\end{abstract}

Keywords: multiple attribute group decision making (MAGDM), interval 2-tuple, plant growth simulation algorithm (PGSA), weighted Steiner point.

DOI: $10.21629 / J S E E .2019 .06 .13$

\section{Introduction}

The multiple attribute group decision-making (MAGDM) problem is an important research topic in complex engineering, economics, and management [1-5]. An expert evaluation matrix is derived through MAGDM analysis, in which each decision maker (DM) considers his/her own preference and provides judgment information on possible alternatives over a range of attributes. Each DM's judgment information is then aggregated to produce an overall ranking of potential alternatives by a given method. Regardless of the utilized method, the primary issue is to aggregate the respective values given by each DM. One of

Manuscript received July 09, 2018.

*Corresponding author.

This work was supported by the National Natural Science Foundation of China (71771118; 71471083), the Ministry of Education Humanities and Social Sciences Foundation of China (18YJCZH146), and the Nanjing University Double First-Class project. the hot research topics is the integration of uncertain linguistic information, which occurs when DMs express their preferences in their native language owing to the nature of the alternative language and their vague knowledge of it. Linguistics is an effective means for humans to express their feelings toward different matters [6,7]. Therefore, a 2-tuple linguistic representation model was proposed to deal with linguistic information [8]. The 2-tuple linguistic representation is composed of a numeric value and a linguistic term and has exact characteristics for linguistic information processing. Moreover, a common problem in linguistic information processing, information distortion and loss, can be effectively avoided with this model $[9,10]$.

Information aggregation takes a key part in the decisionmaking process; thus, a large number of 2-tuple aggregation operators are proposed. Examples include the 2 -tuple weighted averaging operator, the 2-tuple arithmetic mean operator, and the 2-tuple ordered weighted averaging (TOWA) operator [11]; the dependent 2-tuple ordered weighted geometric (DTOWG) operator [12]; the interval TOWA (ITOWA) operator and the interval 2-tuple weighted average (ITWA) operator; the generalized interval 2-tuple weighted average (GITWA) operator, the interval 2-tuple ordered weighted geometric operator, the interval 2-tuple weighted geometric (ITWG) operator, and the generalized interval TOWA operator [13]; the uncertain linguistic hybrid aggregation (ULHA) operator and the uncertain linguistic ordered weighted averaging (ULOWA) operator [14]; and the uncertain linguistic weighted geometric Bonferroni mean (ULWGBM) operator [15]. The main aggregation operators can be categorized into two types, the first of which can transform linguistic assessment information to fuzzy numbers according to the extension principle carried out on the computation and analysis of fuzzy numbers $[16,17]$. The second category calculates and deals with the language phrase symbol directly according to the order and nature of the language evaluation set $[18,19]$. 
Linguistic information collection problems exist and should be improved. Therefore, we propose an optimized interval 2-tuple linguistic aggregation method by using the plant growth simulation algorithm (PGSA) and ITWA operators in order to solve the MAGDM problem in this study. First, we map the attribute value judgment matrix of uncertain linguistic information provided by each expert to the corresponding two-dimensional planar point set. Then, we develop an aggregation operator based on the PGSA, which is used to obtain the general Steiner points (the optimal rally point). Compared with the ant algorithm and the simulated annealing algorithm, the PGSA shows outstanding computational stability in solving the Steiner problem. Employing the optimal number of intervals and the general Steiner points according to the aggregation operator to establish group preference delivers satisfactory simulation results [20-22]. Therefore, the MAGDM method with interval 2-tuple linguistic information on the basis of PGSA and ITWA operators are proposed. In addition, we propose and compare a few dependent interval 2-tuple linguistic aggregation operators in this study. Finally, we present two comparisons to demonstrate that the proposed method is practical and effective. The contributions of this study are characterized by four aspects. First, this study proposes a decision-making method to integrate uncertain linguistic information. Second, the presented method can be used not only to aggregate MAGDM problems but also to solve multi-granularity uncertain linguistic information. Third, the presented method improves the efficiency of uncertain linguistic weighted aggregation (ULWA) characteristics, namely, its high reliability, easy programming, and high-speed calculation to simplify the aggregation and to make it easy to use. Finally, the proposed method combined with a few 2-tuple correlation algorithms has the exact characteristics for linguistic information processing and thus avoiding a common problem in linguistic information processing, i.e., information distortion and loss.

This paper has these following sections: Section 2 introduces a few operation laws and basic concepts of interval 2-tuple linguistic variables; Section 3 develops the PGSA operator to aggregate the expert decision values in the form of interval 2-tuple linguistic variables; Section 4 presents an approach to the MAGDM problem based on PGSA and ITWA operators; Section 5 provides a numerical example to demonstrate the practicality and feasibility of the proposed approaches; Section 6 presents two comparisons; and finally, Section 7 is the conclusion and prospect.

\section{Preliminaries}

\subsection{Linguistic information}

Numerous aspects of distinct activities cannot be assessed quantitatively, but rather qualitatively, that is, with imprecise or vague knowledge. In such cases, an effective approach will not use numerical values, but linguistic assessments. A linguistic variable expresses values in linguistic terms, i.e., the values of a variable are expressed as sentences or words in an artificial or natural language, not as numbers. When traditional quantitative expressions are not suited to reasonably describe too complex or ill-defined situations, linguistic variables will be used.

The basic concept of linguistic information is given as follows: suppose that $S=\left\{s_{i} \mid i=0,1, \ldots, g\right\}$ is a finite and completely ordered discrete term set, where $s_{i}$ denotes the $i$ th linguistic term or label of $s$, and $g+1$ is the cardinality of $s$. Generally, assume $s$ should have the following characteristics [23,24]:

(i) The set is ordered $s_{i}>s_{j}$, if $i>j$;

(ii) The negation operator $\operatorname{Neg}\left(s_{i}\right)=s_{j}$, such that $j=T-i$;

(iii) The maximization operator $\operatorname{Max}\left\{s_{i}, s_{j}\right\}=s_{i}$, if $s_{i} \geqslant s_{j}$;

(iv) The minimization operator Min $\left\{s_{i}, s_{j}\right\}=s_{j}$, if $s_{i} \geqslant s_{j}$.

\subsection{The 2-Tuple linguistic variables}

The 2-tuple is used to characterize the 2-tuple linguistic representation model and to aggregate the linguistic assessment information. By using the proposed method, the commonly occurred information loss and distortion in linguistic variable aggregation can be effectively avoided, and the linguistic assessment information can be precisely judged. The 2-tuple is represented by the 2-tuple $\left(s_{i}, a_{i}\right)$, where $s_{i}$ represents language phrases that belong to the predefined linguistic assessment information set, and $a_{i}$ represents the separation between the calculation and the original language evaluation phrase set [25].

The range of $\beta$ in the 2-tuple linguistic approach is between 0 and $g$, as proposed by Herrera and Martínez. In this case, $\beta$ represents the aggregated result of the evaluation indices of labels which are evaluated with a linguistic term set $S$.

Definition 1 [25] Let $s_{i} \leqslant S$ be a linguistic term. Function $\theta$ is defined as the following formula, which can acquire its corresponding 2-tuple linguistic information:

$$
\left\{\begin{array}{l}
\theta: S \rightarrow S \times[-0.5,0.5) \\
\theta\left(s_{i}\right)=\left(s_{i}, 0\right), \quad s_{i} \in S
\end{array} .\right.
$$

Let $\beta \in[0,1]$ be a value which comes from symbolic aggregation and $S=\left\{s_{o}, s_{1}, \ldots, s_{g}\right\}$ be a linguistic term set. Then, the translation function $\Delta$ can be defined as the following formula, which can acquire the 2-tuple linguistic variable equivalent to $\beta$ :

$$
\Delta:[0, T] \rightarrow S \times[-0.5,0.5),
$$




$$
\Delta(\beta)=\left(s_{i}, \alpha_{i}\right)=\left\{\begin{array}{l}
s_{i}, \quad i=\operatorname{Round}(\beta) \\
\alpha_{i}=\beta-i, \quad \alpha \in[-0.5,0.5)
\end{array}\right.
$$

where $\alpha$ is the value of the symbolic translation, $s_{i}$ has the closest index label to $\beta$, and Round (.) is the usual rounding operation. The number of linguistic terms of $S$ is used to determine the interval of $\alpha$.

Definition 2 [25] Let $\left(s_{i}, \alpha_{i}\right)$ be a 2-tuple linguistic information and $S=\left\{s_{o}, s_{1}, \ldots, s_{g}\right\}$ be a linguistic term set. Then, a function $\Delta^{-1}$ can be used to transform 2-tuple linguistic information into its equivalent numerical value $\beta \in[0,1]$. The function $\Delta^{-1}$ is defined as follows:

$$
\begin{gathered}
\Delta^{-1}: S \times[-0.5,0.5) \rightarrow[0,1] \\
\Delta^{-1}:\left(s_{i}, \alpha_{i}\right)=i+\alpha_{i}=\beta .
\end{gathered}
$$

We can convert linguistic terms into a 2-tuple by adding a 0 value as symbolic translation 1 :

$$
s_{i} \in S \Rightarrow\left(s_{i}, 0\right) \text {. }
$$

Suppose that $\left(s_{i}, \alpha_{i}\right)$ and $\left(s_{j}, \alpha_{j}\right)$ are any two 2-tuples, which possess these properties:

(i) The set is ordered if $i>j:\left(s_{i}, \alpha_{i}\right)>\left(s_{j}, \alpha_{j}\right)$; if $i=j, \alpha_{i}=\alpha_{j}:\left(s_{i}, \alpha_{i}\right)=\left(s_{j}, \alpha_{j}\right)$; if $i=j, \alpha_{i}>\alpha_{j}$ : $\left(s_{i}, \alpha_{i}\right)>\left(s_{j}, \alpha_{j}\right)$.

(ii) The negation operator Neg $\left\{\left(s_{i}, \alpha_{i}\right)\right\}=\Delta\{g-$ $\left.\left[\Delta^{-1}\left(s_{i}, \alpha_{i}\right)\right]\right\}$.

(iii) The maximization operator $\operatorname{Max}\left\{\left(s_{i}, \alpha_{i}\right),\left(s_{j}, \alpha_{j}\right)\right\}$ $=\left(s_{i}, \alpha_{i}\right)$, if $\left(s_{i}, \alpha_{i}\right) \geqslant\left(s_{j}, \alpha_{j}\right)$.

(iv) The minimization operator $\operatorname{Min}\left\{\left(s_{i}, \alpha_{i}\right),\left(s_{j}, \alpha_{j}\right)\right\}$ $=\left(s_{j}, \alpha_{j}\right)$, if $\left(s_{i}, \alpha_{i}\right) \geqslant\left(s_{j}, \alpha_{j}\right)$.

\subsection{Interval number}

We provide preliminaries of interval numbers below to compute uncertain linguistic operators hereinafter. Let $\bar{x}=$ $\left[x^{L}, x^{U}\right]$ be an interval number where $x^{L}, x^{U} \in \mathbf{R}, x^{L} \leqslant$ $x^{U}, \mathbf{R}$ means a real number set. On the other hand, if $x^{L}=x^{U}, \bar{x}$ means a real number, that is, $\bar{x}=x^{L}=x^{U}$. The rules for comparing interval numbers $\bar{x}=\left[x^{L}, x^{U}\right]$ and $\bar{y}=\left[y^{L}, y^{U}\right]$ are as follows:

(i) $\bar{x}>\bar{y}$ if and only if $x^{L}>y^{U}$;

(ii) $\bar{x}=\bar{y}$ if and only if $x^{L}=x^{U}$ and $y^{L}=y^{U}$.

However, if $\bar{x}$ and $\bar{y}$ are overlapped, then it is difficult to determine which one of the two is greater. We present a simple technique to rank interval numbers, wherein an interval can be expressed in the form of a numerical point. This technique can be briefly described as below:

Let $x^{*}$ be the center value of interval $\bar{x}$ and $\nabla x^{*}$ be the error distribution of $\bar{x}$, where $x^{*}=\left(x^{U}+x^{L}\right) / 2$ and $\nabla x^{*}=\left(x^{U}-x^{L}\right) / 2 . \bar{x}$ can be mapped into a crisp value to rank interval numbers by introducing the DMs' risk attitudes, that is, for each interval number, the ranking value can be defined by

$$
\phi_{\varepsilon}(\bar{x})=x^{*}+\varepsilon \nabla x^{*}
$$

where $\varepsilon \in[-1,1]$, and $\varepsilon$ means the optimism/pessimism degree that is used to denote the DMs' risk attitudes. Generally, $\varepsilon$ can be selected by the DM. Depending on the range of $\varepsilon$, DM risk attitudes may include three types, namely, pessimistic, neutral, and optimistic. $\varepsilon$ may have ranges of $-1 \leqslant \varepsilon<0, \varepsilon=0$, and $0<\varepsilon \leqslant 1$. Therefore, the ranking value $\phi_{\varepsilon}(\bar{x})$ may have these corresponding ranges: $\left[x^{*}-\nabla x^{*}, x^{*}\right], x^{*}$ and $\left[x^{*}, x^{*}+\nabla x^{*}\right]$. Obviously, given a specific $\varepsilon, \bar{x}$ is greater than $\bar{y}$, which is denoted as $\bar{x}>\bar{y}$ if and only if $\phi_{\varepsilon}(\bar{x})>\phi_{\varepsilon}(\bar{y}) . \bar{x}$ is equal to $\bar{y}$, which is denoted as $\bar{x}=\bar{y}$ if and only if $\phi_{\varepsilon}(\bar{x})=\phi_{\varepsilon}(\bar{y})$.

\subsection{Interval 2-tuple linguistic variables}

The model of interval 2-tuple linguistic representation is now widely applied and studied, including material and personnel selection, complex engineering, economics, management, and robot evaluation. This model has the following definitions:

Definition 3 [26] Let $S=\left\{s_{0}, s_{1}, \ldots, s_{g}\right\}$ be a linguistic term set, in which two linguistic terms form an interval linguistic variable, which is denoted by $\left[s_{i}, s_{j}\right]$, where $i \leqslant j$, and $s_{i}, s_{j}$ mean the linguistic terms of the set $S$, and $s_{i}$ and $s_{j}$ mean respectively lower and upper limits.

Definition 4 [27] Let $S=\left\{s_{0}, s_{1}, \ldots, s_{g}\right\}$ be a linguistic term set, in which two 2-tuples form an interval 2-tuple linguistic variable, which are denoted by $\left[\left(s_{i}, a_{i}\right),\left(s_{j}, a_{j}\right)\right]$, where $i \leqslant j$ and $a_{i} \leqslant a_{j}, s_{i}\left(s_{j}\right)$ and $a_{i}\left(a_{j}\right)$ mean linguistic labels in the term set $S$ and the symbolic translation, respectively. The interval 2-tuple which transforms equivalent information to an interval number $\left[\beta_{1}, \beta_{2}\right]\left(\beta_{1}, \beta_{2} \in[0,1], \beta_{1}<\beta_{2}\right)$ is derived with this function

$$
\begin{gathered}
\Delta\left[\beta_{1}, \beta_{2}\right]=\left[\left(s_{i}, a_{i}\right),\left(s_{j}, a_{j}\right)\right] \\
\left\{\begin{array}{l}
s_{i}, \quad i=\operatorname{Round}\left(\beta_{1}, g\right) \\
s_{j}, \quad j=\operatorname{Round}\left(\beta_{2}, g\right) \\
a_{i}=\beta_{1}-i, \quad a_{i} \in[-0.5,0.5) \\
a_{j}=\beta_{2}-j, \quad a_{j} \in[-0.5,0.5)
\end{array}\right.
\end{gathered}
$$

On the contrary, there is always a function which can be used to convert an interval 2-tuple into an interval number $\left[\beta_{1}, \beta_{2}\right]\left(\beta_{1}, \beta_{2} \in[0,1], \beta_{1}<\beta_{2}\right)$ as follows:

$$
\Delta^{-1}\left[\left(s_{i}, a_{i}\right),\left(s_{j}, a_{j}\right)\right]=\left[i+a_{i}, j+a_{j}\right]=\left[\beta_{1}, \beta_{2}\right] .
$$

Specifically, the condition of $s_{i}=s_{j}$ and $a_{i}=a_{j}$ will decrease the interval 2-tuple linguistic variable down to a 2-tuple linguistic variable. 


\section{The PGSA and ITWA operators}

\subsection{The ITWA operator}

Definition 5 [27] Let $\widetilde{a}_{i}=\left[\left(r_{i}, a_{i}\right),\left(t_{i}, \varepsilon_{i}\right)\right](i=$ $1,2, \ldots, n)$ be a set of interval 2-tuples and $\boldsymbol{w}=$ $\left\{w_{0}, w_{1}, \ldots, w_{n}\right\}^{\mathrm{T}}$ be their associated weight, where $w_{i} \in$ $[0,1], \sum_{i=1}^{n} w_{i}=1$. The ITWA operator can be expressed by

$$
\begin{gathered}
\operatorname{ITWA}_{\boldsymbol{w}}\left(\widetilde{a}_{1}, \widetilde{a}_{2}, \ldots, \widetilde{a}_{n}\right)=\sum_{i=1}^{n} w_{i} \widetilde{a}_{i}= \\
\Delta\left[\sum_{t=1}^{n} w_{i} \Delta^{-1}\left(r_{i}, a_{i}\right), \sum_{i=1}^{n} w_{i} \Delta^{-1}\left(t_{i}, \varepsilon_{i}\right)\right] .
\end{gathered}
$$

\subsection{Obtaining the optimal aggregation evaluation value based on the PGSA}

\subsubsection{Mapping of uncertain linguistic information in} decision matrices

Let interval linguistic variable $\left[s_{a^{i j}}^{(t)}, s_{b^{i j}}^{(t)}\right] \quad(i=$ $1,2, \ldots, m ; j=1,2, \ldots, n ; t=1,2, \ldots, p), s_{a^{i j}}^{(t)}, s_{b^{i j}}^{(t)} \in$ $S, a^{i j}, b^{i j} \in\{0,1, \ldots, T\}, a^{i j} \leqslant b^{i j}$. Thus $\widetilde{S}_{i j}=$ $\left[\left(s_{a^{i j}}^{(t)}, 0\right),\left(s_{b^{i j}}^{(t)}, 0\right)\right]$ is the interval 2-tuple. According to Definition $4, \Delta^{-1}\left(\widetilde{S}_{i j}\right)=\left[\Delta^{-1}\left(s_{a^{i j}}^{(t)}, 0\right), \Delta^{-1}\left(s_{b^{i j}}^{(t)}, 0\right)\right]$ can be obtained, which is expressed as random interval numbers $\boldsymbol{\beta}^{(t)}=\left[\beta_{a^{i j}}^{(t)}, \beta_{b^{i j}}^{(t)}\right]_{m \times n}$. Then, the interval numbers are mapped into two-dimensional coordinates corresponding to the decision preference information where $\beta_{a^{i j}}^{(t)}$ is the abscissa value and $\beta_{b^{i j}}^{(t)}$ is the ordinate value. Therefore, an MAGDM problem with interval numbers that contain $t$ DMs and $m$ alternatives concerning $n$ attributes may be seen as $p$ planes that are composed of $m \times n$ planar point sets

$$
\begin{aligned}
& \boldsymbol{\beta}^{(t)}=\left[\beta_{a^{i j}}^{(t)}, \beta_{b^{i j}}^{(t)}\right]_{m \times n} \rightarrow\left(\beta_{a^{i j}}^{(t)}, \beta_{b^{i j}}^{(t)}\right) \in \mathbf{R}^{2}, \\
& i=1,2, \ldots, m ; j=1,2, \ldots, n ; t=1,2, \ldots, p
\end{aligned}
$$

where $\mathbf{R}^{2}$ expresses the two-dimensional Euclidean space.

\subsubsection{Aggregation of the uncertain linguistic information decision matrices}

We map the uncertain linguistic information into a planar point set. The aggregation of expert evaluation is needed to find an assembly point that best represents the evaluation points. If the sum of the distances between one and several other points is the shortest, then this rally point can best represent the other points. The corresponding interval 2-tuple of this rally point is the most comprehensive evaluation value that represents the evaluation value of different experts. Based on the initial definition of the Fermat point by the mathematician Fermat, for any given point on the planes $p_{1}, p_{2}, p_{3}$, find another point $P$ and connect point $P$ with points $p_{1}, p_{2}, p_{3}$. The minimum distance of the point of attachment is known mathematically as the "Fermat problem," and point $P$ is called the "Fermat point" [28]. In the beginning of the 19th century, this problem was further extended by Swiss mathematician Steiner to allow the plane to include a finite number of points, i.e., a unique point $P$ is found to obtain the minimal total Euclidean distance [29]. A natural extension of the Steiner problem may be expressed by the difinition as follows.

Definition 6 Based on the $n$ points $p_{1}, p_{2}, \ldots, p_{n}(n \geqslant$ 4 ) on the plane, if there is a point $P$ that minimizes the distance $D=\sum_{i=1}^{n} w_{i}\left|P P_{i}\right|$ where the corresponding positive weights $w_{i} \in[0,1](i=1,2, \ldots, n)$, then this is called a weighted Steiner problem. Thus, we call point $P$ the weighted Steiner point (see Fig. 1).

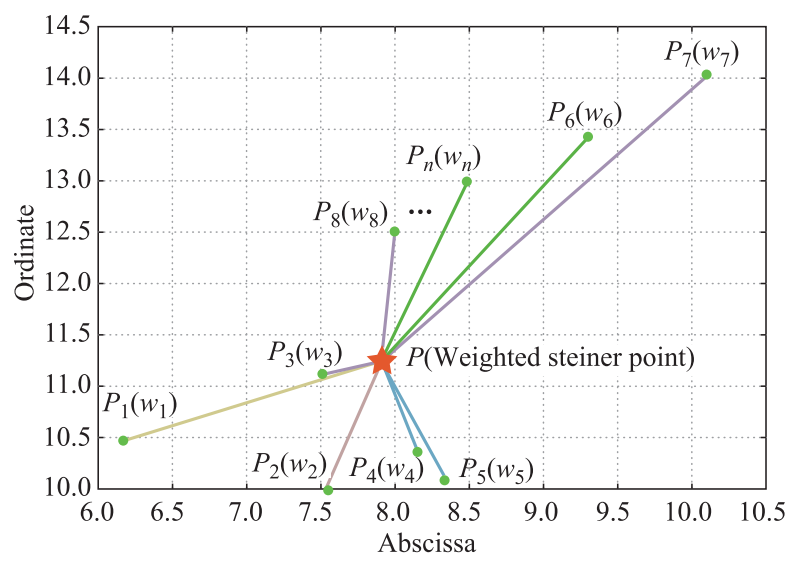

Fig. 1 Weighted Steiner problem

According to Section 3.1, we can map the attribute value judgment matrix of uncertain linguistic variables on twodimensional planar point sets, that is, $\bar{\beta}^{(t)} \rightarrow \mathbf{R}^{2}(t=$ $1,2, \ldots, n)$. Thus, the aggregation on the interval number $\left[\beta_{a^{i j}}^{(t)}, \beta_{b^{i j}}^{(t)}\right]$ is the equivalent of the set in the plane $e_{1}, e_{2}, \ldots, e_{n}(n>3)$. Obviously, we should find a point $E^{*}$ where the distance of the point of attachment is the minimum.

\subsubsection{Obtaining the generalized Steiner point of the expert evaluation points by using PGSA}

Unfortunately, $P$ cannot be obtained by elementary geometry methods, and the difficulty increases as the known points increase. We use the PGSA to find the optimal assembly point $P$ to solve this problem.

The PGSA, which is a kind of intelligent optimization algorithm based on the principle of plant to light, was first proposed by Li [30], which has a simple parameter set- 
ting, fewer restrictions, strong random searching ability, and high stability; thus, it is superior in solving global optimal solutions and has been widely used in numerous research fields in recent years [31-35]. Scholars have compared the PGSA with the genetic algorithm (GA) and the particle swarm optimization algorithm, and the results in the existing literature show that the optimal network proposed by the PGSA is a better scheme [33]. According to PGSA scholars, theoretical analyses and numerical example results have shown that the PGSA has certain advantages compared with the GA as a representative of the modern heuristic algorithm. For example, the PGSA can be treated as the objective function and the constraint condition separately with no encoding and decoding, it can avoid constructing new calculating objective functions, and it does not encounter the problems of punishment coefficients, such as selection, crossover and mutation rates, and satisfactory stability of solutions. The PGSA has an ideal search mechanism with directional and random equilibrium, which is determined by the morphogen concentration, and can find the global optimal solution at a relatively fast speed [29,34]. The procedure proposed to obtain the generalized Fermat point is as follows:

Step 1 To determine the initial growing point, $a_{m} \in X$. $X$ is the length of the bounded closed box of $\mathbf{R}^{n}$ with initial growing point $a_{m}$ as the uniform random point in the bounded closed box.

Step 2 To determine the rate of the growing point,

$$
p_{m}=\frac{\sum_{i=1}^{n}\left(1 /\left|a_{m} s_{i}\right|\right)}{\sum_{m=1}^{v} \sum_{i=1}^{n}\left(1 /\left|a_{m} s_{i}\right|\right)}, \quad m=1,2, \ldots, v .
$$

Step 3 To establish the interval probability of the growing point on the closed space according to the result of Step 2. Then, select the current iteration growing point by random numbers.

Step 4 To determine the step length $\lambda$ (take $\lambda$ as $l / 1000)$, let the growing point $a_{m}$ grow according to the system $L$ with $\partial=90^{\circ}$. Then, replace the point $a_{m}$ with the new growth rally points.

Step 5 If new growing points are no longer produced and the preset number of iterations have been reached, obtain the global optimal and local optimal solutions and stop computing; otherwise, return to Step 2.

\section{An approach to MAGDM}

In this section, the attribute preference values are the form of interval 2-tuple linguistic variables, while the attribute and the expert weights take the form of real numbers.
Let $S=\left\{s_{0}, s_{1}, \ldots, s_{T}\right\}$ be a linguistic term of $T+1$ and $S$ be a set of interval 2-tuple linguistic variables. Let $X=\left\{X_{1}, X_{2}, \ldots, X_{m}\right\}(m \geqslant 2)$ be a set of $m$ discrete alternatives and $u=\left\{u_{1}, u_{2}, \ldots, u_{n}\right\}(n \geqslant 2)$ be a set of attributes. Let $D=\left\{d_{1}, d_{2}, \ldots, d_{l}\right\}(l \geqslant 2)$ be a set of $l \mathrm{DMs}$ $\operatorname{DM}_{k}(k=1,2, \ldots, l)$ and $\boldsymbol{w}=\left\{w_{1}, w_{2}, \ldots, w_{n}\right\}^{\mathrm{T}}$ be a weight vector of attributes. Suppose that $\boldsymbol{D}^{k}=\left[\bar{d}_{i j}^{k}\right]_{m \times n}$ is a linguistic decision matrix of interval 2-tuple, where $\bar{d}_{i j}^{k}$ is the attribute value for alternative $x_{i}$ concerning attribute $u_{i}$, which is provided by DM $d_{k}$ and is in the format of interval 2-tuple linguistic variables $\bar{d}_{i j}^{k} \in \bar{S}$. In addition, DMs can utilize various linguistic term sets for expressing the preference values.

This paper uses the MAGDM problem to rank the alternatives or to select the most desirable one(s) among a finite set $X$ by using the interval 2-tuple linguistic decision matrix $\boldsymbol{D}^{k}=\left[\bar{d}_{i j}^{k}\right]_{m \times n}$. An approach is proposed here to solve this problem with interval 2-tuple linguistic variables by means of the PGSA operator. The methods include these steps:

Step 1 Convert the linguistic decision matrix $\boldsymbol{D}^{k}=$ $\left[\bar{d}_{i j}^{k}\right]_{m \times n}$ into the linguistic decision matrix of interval 2 tuple $\left.\widetilde{\boldsymbol{R}}_{k}=\left(\widetilde{r}_{i j}\right)_{m \times n}=\left[\left(r_{i j}^{k}, 0\right),\left(t_{i j}^{k}, 0\right)\right]\right)_{m \times n}$, where $r_{i j}^{k}, t_{i j}^{k} \in S, S=\left\{s_{i} \mid i=0,1,2, \ldots, g\right\}$ and $r_{i j}^{k} \leqslant t_{i j}^{k}$.

Suppose that $\mathrm{DM}_{k}$ provides its assessment on a set of five linguistic terms, where the linguistic term set is defined as

$$
\begin{gathered}
S=\left\{s_{0}=\text { very poor, } s_{1}=\text { poor }, s_{2}=\right.\text { slightly poor } \\
s_{3}=\text { fair, } s_{4}=\text { slightly good, } s_{5}=\text { good } \\
\left.s_{6}=\text { very good }\right\}
\end{gathered}
$$

The following requirements can be used to convert the linguistic information contained in the decision matrix into its corresponding interval 2-tuple linguistic assessment: (i) a certain grade, e.g., poor can be written as $\left[\left(s_{1}, 0\right),\left(s_{1}, 0\right)\right]$ and (ii) e.g., poor-medium means that the attribute under consideration is between poor and medium, which may be defined as $\left[\left(s_{1}, 0\right),\left(s_{3}, 0\right)\right]$.

Step 2 Utilize the decision information contained in matrix $\widetilde{\boldsymbol{R}}_{k}$ and the ITWA operator

$$
\begin{gathered}
\widetilde{r}_{i}^{k}=\left[\left(r_{i}^{k}, a_{i}^{k}\right),\left(t_{i}^{k}, \varepsilon_{i}^{k}\right)\right]= \\
\operatorname{ITWA}_{\boldsymbol{w}}\left(\widetilde{r}_{i 1}^{k}, \widetilde{r}_{i 2}^{k}, \ldots, \widetilde{r}_{i m}^{k}\right)= \\
\Delta\left[\sum_{j=1}^{n} w_{j} \Delta^{-1}\left(r_{i j}^{k}, 0\right), \sum_{j=1}^{n} w_{j} \Delta^{-1}\left(t_{i j}^{k}, \varepsilon_{i}\right)\right], \\
i=1,2, \ldots, m ; k=1,2, \ldots, l
\end{gathered}
$$

to derive the individual overall preference values $\widetilde{r}_{k}(k=$ $1,2, \ldots, 5)$ of the alternative $A_{i}$. 
Step 3 Map the attribute value judgment matrix of uncertain linguistic variables to two-dimensional planar point sets according to the fourth part. We can obtain $\boldsymbol{\beta}^{(t)}=$ $\left[\beta_{a^{i j}}^{(t)}, \beta_{b^{i j}}^{(t)}\right]_{m \times n}$.

Step 4 Utilize the decision information contained in the matrix and aggregate all the DMs' opinions based on the PGSA into a collective alternative appraisal value to get the optimal interval numbers.

Step 5 Rank the alternatives or select the most desirable one(s) according to the comparison laws of (6).

\section{Illustrative example}

\subsection{Problem description and the decision process}

We present an MAGDM example of a robot selection problem in this section. After the preliminary simulation, we select five robots (alternatives) $A_{i}(i=1,2, \ldots, 5)$ with the capacity to perform the chosen task. A committee of four DMs, that is, $\mathrm{DM}_{1}, \mathrm{DM}_{2}, \mathrm{DM}_{3}, \mathrm{DM}_{4}$ are created to determine the optimum robot (alternative) for the application. The analysis includes these alternative attributes: $C_{1}, C_{2}, C_{3}$ (purchase cost), $C_{4}$ and $C_{5}$. The attribute weight vectors are $\boldsymbol{w}=(0.18,0.30,0.18,0.12,0.22)^{\mathrm{T}}$. Each DM will consider its own preference and provide its judgment (uncertain linguistic term) on possible alternatives over multiple attributes. Then, we can get an expert evaluation matrix. We need to aggregate each DMs' judgment information to obtain an overall ranking of the five alternatives. Specifically, $\mathrm{DM}_{1}$ will provide the judgement by means of linguistic term set $A, \mathrm{DM}_{2}$ will provide by means of $B, \mathrm{DM}_{3}$ will provide by means of $C$, and $\mathrm{DM}_{4}$ will provide by means of $D$. These linguistic term sets are defined according to the following formulas:

$$
A=\left\{a_{0}=\text { very low, } a_{1}=\text { low }, a_{2}=\right.\text { medium, }
$$

$$
\begin{gathered}
\left.a_{3}=\text { high }, a_{4}=\text { very high }\right\}, \\
B=\left\{b_{0}=\text { very low, } b_{1}=\text { low },\right.
\end{gathered}
$$

$b_{2}=$ medium low,$b_{3}=$ medium,$b_{4}=$ medium high,

$$
\begin{gathered}
\left.b_{5}=\text { high, } b_{6}=\text { very high }\right\} \\
C=\left\{c_{0}=\text { extra low, } c_{1}=\text { very low, } c_{2}=\text { low },\right.
\end{gathered}
$$$$
c_{3}=\text { medium low }, c_{4}=\text { medium, } c_{5}=\text { medium high, }
$$

\begin{tabular}{|c|c|c|c|c|c|c|}
\hline \multirow{2}{*}{ DM } & \multirow{2}{*}{ Alternative } & \multicolumn{5}{|c|}{ Attribute } \\
\hline & & $C_{1}$ & $C_{2}$ & $C_{3}$ & $C_{4}$ & $C_{5}$ \\
\hline \multirow{5}{*}{$\mathrm{DM}_{1}$} & $A_{1}$ & $\mathrm{M}$ & M-H & $\mathrm{H}$ & $\mathrm{H}$ & $\mathrm{M}$ \\
\hline & $A_{2}$ & M-H & M & $\mathrm{H}$ & M & M-H \\
\hline & $A_{3}$ & $\mathrm{H}$ & $\mathrm{H}-\mathrm{VH}$ & $\mathrm{VH}$ & $\mathrm{H}$ & $\mathrm{VH}$ \\
\hline & $A_{4}$ & M & M & L-M & $\mathrm{L}$ & $\mathrm{L}$ \\
\hline & $A_{5}$ & $\mathrm{H}$ & $\mathrm{H}-\mathrm{VH}$ & M & $\mathrm{H}$ & $\mathrm{H}$ \\
\hline \multirow{5}{*}{$\mathrm{DM}_{2}$} & $A_{1}$ & $\bar{M}$ & $\mathrm{M}$ & ML-M & $\mathrm{MH}$ & M-H \\
\hline & $A_{2}$ & ML-M & M & $\mathrm{L}$ & M & ML \\
\hline & $A_{3}$ & $\mathrm{H}$ & VH & $\mathrm{H}$ & VH & $\mathrm{VH}$ \\
\hline & $A_{4}$ & $\mathrm{~L}$ & MH & M & ML & ML \\
\hline & $A_{5}$ & M-H & $\mathrm{H}$ & $\mathrm{H}$ & MH-H & $\mathrm{H}$ \\
\hline \multirow{5}{*}{$\mathrm{DM}_{3}$} & $A_{1}$ & $\mathrm{M}$ & $\mathrm{M}$ & $\mathrm{H}-\mathrm{VH}$ & $\mathrm{H}$ & M-H \\
\hline & $A_{2}$ & M-MH & M-H & $\mathrm{H}$ & $\mathrm{H}$ & $\mathrm{H}$ \\
\hline & $A_{3}$ & EH & $\mathrm{VH}$ & VH-EH & VH & $\mathrm{VH}$ \\
\hline & $A_{4}$ & $\mathrm{~L}$ & ML & $\mathrm{L}$ & L-ML & L-M \\
\hline & $A_{5}$ & VH & $\mathrm{VH}$ & $\mathrm{H}-\mathrm{VH}$ & $\mathrm{H}-\mathrm{VH}$ & $\mathrm{VH}$ \\
\hline \multirow{5}{*}{$\mathrm{DM}_{4}$} & $A_{1}$ & M & M & $\mathrm{H}$ & $\mathrm{H}$ & M-H \\
\hline & $A_{2}$ & M-H & M & $\mathrm{H}$ & $\mathrm{H}$ & $\mathrm{H}$ \\
\hline & $A_{3}$ & $\mathrm{H}$ & $\mathrm{VH}$ & $\mathrm{H}-\mathrm{VH}$ & VH & $\mathrm{VH}$ \\
\hline & $A_{4}$ & L-M & M & $\mathrm{L}$ & L-M & $\mathrm{M}$ \\
\hline & $A_{5}$ & $\mathrm{H}$ & $\mathrm{VH}$ & $\mathrm{H}$ & H-VH & $\mathrm{H}$ \\
\hline
\end{tabular}

$$
\begin{gathered}
\left.c_{6}=\text { high }, c_{7}=\text { very high, } c_{8}=\text { extra high }\right\}, \\
D=\left\{d_{0}=\text { very low, } d_{1}=\text { low, } d_{2}=\right.\text { medium, } \\
\left.d_{3}=\text { high, } d_{4}=\text { very high }\right\} .
\end{gathered}
$$

\begin{tabular}{|c|c|c|c|c|c|c|}
\hline \multirow{2}{*}{$\mathrm{DM}$} & \multirow{2}{*}{ Alternative } & \multicolumn{5}{|c|}{ Attribute } \\
\hline & & $C_{1}$ & $C_{2}$ & $C_{3}$ & $C_{4}$ & $C_{5}$ \\
\hline \multirow{5}{*}{$\mathrm{DM}_{1}$} & $A_{1}$ & {$\left[\left(a_{2}, 0\right),\left(a_{2}, 0\right)\right]$} & {$\left[\left(a_{2}, 0\right),\left(a_{3}, 0\right)\right]$} & {$\left[\left(a_{2}, 0\right),\left(a_{3}, 0\right)\right]$} & {$\left[\left(a_{3}, 0\right),\left(a_{3}, 0\right)\right]$} & {$\left[\left(a_{2}, 0\right),\left(a_{2}, 0\right)\right]$} \\
\hline & $A_{2}$ & {$\left[\left(a_{2}, 0\right),\left(a_{3}, 0\right)\right]$} & {$\left[\left(a_{2}, 0\right),\left(a_{2}, 0\right)\right]$} & {$\left[\left(a_{3}, 0\right),\left(a_{3}, 0\right)\right]$} & {$\left[\left(a_{2}, 0\right),\left(a_{2}, 0\right)\right]$} & {$\left[\left(a_{2}, 0\right),\left(a_{3}, 0\right)\right]$} \\
\hline & $A_{3}$ & {$\left[\left(a_{3}, 0\right),\left(a_{3}, 0\right)\right]$} & {$\left[\left(a_{3}, 0\right),\left(a_{4}, 0\right)\right]$} & {$\left[\left(a_{4}, 0\right),\left(a_{4}, 0\right)\right]$} & {$\left[\left(a_{3}, 0\right),\left(a_{3}, 0\right)\right]$} & {$\left[\left(a_{4}, 0\right),\left(a_{4}, 0\right)\right]$} \\
\hline & $A_{4}$ & {$\left[\left(a_{2}, 0\right),\left(a_{2}, 0\right)\right]$} & {$\left[\left(a_{2}, 0\right),\left(a_{3}, 0\right)\right]$} & {$\left[\left(a_{1}, 0\right),\left(a_{2}, 0\right)\right]$} & {$\left[\left(a_{1}, 0\right),\left(a_{1}, 0\right)\right]$} & {$\left[\left(a_{1}, 0\right),\left(a_{1}, 0\right)\right]$} \\
\hline & $A_{5}$ & {$\left[\left(a_{3}, 0\right),\left(a_{3}, 0\right)\right]$} & {$\left[\left(a_{3}, 0\right),\left(a_{4}, 0\right)\right]$} & {$\left[\left(a_{2}, 0\right),\left(a_{2}, 0\right)\right]$} & {$\left[\left(a_{3}, 0\right),\left(a_{3}, 0\right)\right]$} & {$\left[\left(a_{3}, 0\right),\left(a_{3}, 0\right)\right]$} \\
\hline \multirow{4}{*}{$\mathrm{DM}_{2}$} & $A_{2}$ & {$\left[\left(b_{2}, 0\right),\left(b_{3}, 0\right)\right]$} & {$\left[\left(b_{3}, 0\right),\left(b_{3}, 0\right)\right]$} & {$\left[\left(b_{1}, 0\right),\left(b_{1}, 0\right)\right]$} & {$\left[\left(b_{3}, 0\right),\left(b_{3}, 0\right)\right]$} & {$\left[\left(b_{2}, 0\right),\left(b_{2}, 0\right)\right]$} \\
\hline & $A_{3}$ & {$\left[\left(b_{5}, 0\right),\left(b_{5}, 0\right)\right]$} & {$\left[\left(b_{5}, 0\right),\left(b_{5}, 0\right)\right]$} & {$\left[\left(b_{5}, 0\right),\left(b_{5}, 0\right)\right]$} & {$\left[\left(b_{5}, 0\right),\left(b_{5}, 0\right)\right]$} & {$\left[\left(b_{5}, 0\right),\left(b_{5}, 0\right)\right]$} \\
\hline & $A_{4}$ & {$\left[\left(b_{1}, 0\right),\left(b_{1}, 0\right)\right]$} & {$\left[\left(b_{4}, 0\right),\left(b_{4}, 0\right)\right]$} & {$\left[\left(b_{3}, 0\right),\left(b_{3}, 0\right)\right]$} & {$\left[\left(b_{2}, 0\right),\left(b_{2}, 0\right)\right]$} & {$\left[\left(b_{2}, 0\right),\left(b_{2}, 0\right)\right]$} \\
\hline & $A_{5}$ & {$\left[\left(b_{3}, 0\right),\left(b_{5}, 0\right)\right]$} & {$\left[\left(b_{5}, 0\right),\left(b_{5}, 0\right)\right]$} & {$\left[\left(b_{5}, 0\right),\left(b_{5}, 0\right)\right]$} & {$\left[\left(b_{4}, 0\right),\left(b_{5}, 0\right)\right]$} & {$\left[\left(b_{5}, 0\right),\left(b_{5}, 0\right)\right]$} \\
\hline \multirow{4}{*}{$\mathrm{DM}_{3}$} & $A_{1}$ & {$\left[\left(c_{4}, 0\right),\left(c_{4}, 0\right)\right]$} & {$\left[\left(c_{4}, 0\right),\left(c_{4}, 0\right)\right]$} & {$\left[\left(c_{6}, 0\right),\left(c_{7}, 0\right)\right]$} & {$\left[\left(c_{6}, 0\right),\left(c_{6}, 0\right)\right]$} & {$\left[\left(c_{4}, 0\right),\left(c_{6}, 0\right)\right]$} \\
\hline & $A_{2}$ & {$\left[\left(c_{4}, 0\right),\left(c_{5}, 0\right)\right]$} & {$\left[\left(c_{4}, 0\right),\left(c_{6}, 0\right)\right]$} & {$\left[\left(c_{6}, 0\right),\left(c_{6}, 0\right)\right]$} & {$\left[\left(c_{6}, 0\right),\left(c_{6}, 0\right)\right]$} & {$\left[\left(c_{6}, 0\right),\left(c_{6}, 0\right)\right]$} \\
\hline & $A_{4}$ & {$\left[\left(c_{2}, 0\right),\left(c_{2}, 0\right)\right]$} & {$\left[\left(c_{3}, 0\right),\left(c_{3}, 0\right)\right]$} & {$\left[\left(c_{2}, 0\right),\left(c_{2}, 0\right)\right]$} & {$\left[\left(c_{2}, 0\right),\left(c_{3}, 0\right)\right]$} & {$\left[\left(c_{2}, 0\right),\left(c_{4}, 0\right)\right]$} \\
\hline & $A_{5}$ & {$\left[\left(c_{7}, 0\right),\left(c_{7}, 0\right)\right]$} & {$\left[\left(c_{7}, 0\right),\left(c_{7}, 0\right)\right]$} & {$\left[\left(c_{6}, 0\right),\left(c_{7}, 0\right)\right]$} & {$\left[\left(c_{6}, 0\right),\left(c_{7}, 0\right)\right]$} & {$\left[\left(c_{7}, 0\right),\left(c_{7}, 0\right)\right]$} \\
\hline
\end{tabular}

Table 1 presents how the four DMs assess the five alternatives on each of the attributes.

Table 1 Linguistic assessments

Table 2 Interval 2-tuple linguistic decision matrix of the DMs 
Continued

\begin{tabular}{ccccccc}
\hline \multirow{2}{*}{ DM } & \multirow{2}{*}{ Alternative } & \multicolumn{4}{c}{ Attribute } \\
\cline { 2 - 6 } & & $C_{1}$ & $C_{2}$ & $C_{3}$ & $C_{4}$ & $C_{5}$ \\
\hline \multirow{4}{*}{$A_{1}$} & {$\left[\left(d_{2}, 0\right),\left(d_{2}, 0\right)\right]$} & {$\left[\left(d_{2}, 0\right),\left(d_{2}, 0\right)\right]$} & {$\left[\left(d_{3}, 0\right),\left(d_{3}, 0\right)\right]$} & {$\left[\left(d_{3}, 0\right),\left(d_{3}, 0\right)\right]$} & {$\left[\left(d_{2}, 0\right),\left(d_{3}, 0\right)\right]$} \\
& $A_{2}$ & {$\left[\left(d_{2}, 0\right),\left(d_{3}, 0\right)\right]$} & {$\left[\left(d_{2}, 0\right),\left(d_{2}, 0\right)\right]$} & {$\left[\left(d_{3}, 0\right),\left(d_{3}, 0\right)\right]$} & {$\left[\left(d_{3}, 0\right),\left(d_{3}, 0\right)\right]$} & {$\left[\left(d_{3}, 0\right),\left(d_{3}, 0\right)\right]$} \\
& $A_{3}$ & {$\left[\left(d_{3}, 0\right),\left(d_{3}, 0\right)\right]$} & {$\left[\left(d_{4}, 0\right),\left(d_{4}, 0\right)\right]$} & {$\left[\left(d_{3}, 0\right),\left(d_{4}, 0\right)\right]$} & {$\left[\left(d_{4}, 0\right),\left(d_{4}, 0\right)\right]$} & {$\left[\left(d_{4}, 0\right),\left(d_{4}, 0\right)\right]$} \\
& $A_{4}$ & {$\left[\left(d_{1}, 0\right),\left(d_{2}, 0\right)\right]$} & {$\left[\left(d_{2}, 0\right),\left(d_{2}, 0\right)\right]$} & {$\left[\left(d_{1}, 0\right),\left(d_{1}, 0\right)\right]$} & {$\left[\left(d_{1}, 0\right),\left(d_{2}, 0\right)\right]$} & {$\left[\left(d_{2}, 0\right),\left(d_{2}, 0\right)\right]$} \\
& $A_{5}$ & {$\left[\left(d_{3}, 0\right),\left(d_{3}, 0\right)\right]$} & {$\left[\left(d_{4}, 0\right),\left(d_{4}, 0\right)\right]$} & {$\left[\left(d_{3}, 0\right),\left(d_{3}, 0\right)\right]$} & {$\left[\left(d_{3}, 0\right),\left(d_{4}, 0\right)\right]$} & {$\left[\left(d_{3}, 0\right),\left(d_{3}, 0\right)\right]$} \\
\hline
\end{tabular}

The overall ranking of the five alternatives is obtained by using the methods proposed in this paper, after which the most desirable alternative can be accordingly obtained. The steps are as follows:

Step 1 The decision matrix presented in Table 1 is converted into the linguistic decision matrix of interval 2-tuple $\widetilde{\boldsymbol{R}}_{k}=\left(\widetilde{r}_{i j}\right)_{m \times n}=\left(\left[\left(r_{i j}^{k}, 0\right),\left(t_{i j}^{k}, 0\right)\right]\right)_{m \times n}$, as is depicted in Table 2.

Step 2 The individual overall preference values $\widetilde{r}_{k}(k=$ $1,2, \ldots, 5)$ of the alternative $A_{i}$ are derived by means of the decision information contained in matrix $\widetilde{\boldsymbol{R}}_{k}$ and the ITWA operator.

Step 3 Map the attribute value judgment matrix of the uncertain linguistic variable on two-dimensional planar point sets according to the fourth part. We can obtain $\boldsymbol{\beta}^{(t)}=\left[\beta_{a^{i j}}^{(t)}, \beta_{b^{i j}}^{(t)}\right]_{m \times n}($ refer to Table 3$)$.
Step 4 Utilize the decision information given in Table 3. Aggregate all the DMs' opinions based on the PGSA into a collective alternative appraisal value to get the optimal interval numbers (see Table 4).

Table 3 Individual overall preference values indicated by $\left[\boldsymbol{\beta}_{1}, \boldsymbol{\beta}_{2}\right]$

\begin{tabular}{lccccc}
\hline \multirow{2}{*}{$\mathrm{DM}$} & \multicolumn{5}{c}{ Alternative } \\
\cline { 2 - 6 } & $A_{1}$ & $A_{2}$ & $A_{3}$ & $A_{4}$ & $A_{5}$ \\
\hline $\mathrm{DM}_{1}$ & {$[4.60,5.20]$} & {$[4.36,5.16]$} & {$[6.80,7.40]$} & {$[2.96,3.32]$} & {$[5.64,6.24]$} \\
$\mathrm{DM}_{2}$ & {$[3.92,4.74]$} & {$[2.98,3.22]$} & {$[7.52,7.52]$} & {$[3.47,3.47]$} & {$[6.02,6.67]$} \\
$\mathrm{DM}_{3}$ & {$[4.60,5.22]$} & {$[5.04,5.82]$} & {$[7.18,7.36]$} & {$[2.30,2.86]$} & {$[6.70,7.00]$} \\
$\mathrm{DM}_{4}$ & {$[4.60,5.04]$} & {$[5.04,5.40]$} & {$[7.28,7.64]$} & {$[3.04,3.64]$} & {$[6.60,6.84]$} \\
\hline
\end{tabular}

Step 5 Rank the alternatives or select the most desirable one(s) according to the comparison laws of different aggregation methods. The ranking orders of the alternatives are $A_{3} \succ A_{5} \succ A_{2} \succ A_{1} \succ A_{4}$ and the best alternative is $A_{3}$. $\succ$ means "preferred to".

Table 4 Optimal interval numbers by PGSA

\begin{tabular}{|c|c|c|c|c|c|}
\hline Alternative & $\overline{A_{1}}$ & $\overline{A_{2}}$ & $\overline{A_{3}}$ & $\overline{A_{4}}$ & $\overline{A_{5}}$ \\
\hline The optimal interval number by PGSA & {$[4.600,5.200]$} & {$[4.578,5.237]$} & {$[7.219,7.470]$} & {$[2.960,3.320]$} & {$[6.392,6.779]$} \\
\hline The ranking value $\phi$ & 4.780 & 4.776 & 7.294 & 3.068 & 6.058 \\
\hline
\end{tabular}

\subsection{Comparison with other operators}

We obtain the optimal interval numbers based on the PGSA by utilizing the decision information given in Table 3 . Then, we aggregate all the DMs' opinions by using the air mean operator, the ITWG operator, and the depended interval 2-tuple weighted average (DITWA) operator, which are shown in Table 5. In addition, we count the sum of the distance of the assembly point and the other points by using different aggregation methods. Their comparison is depicted in Table 6. The ranking order of the alternatives are shown in Table 7. The ranking order of the alternative robots may differ depending on the aggregation operators used. Therefore, the results may lead to different decisions depending on the particular type of the utilized aggregation operator. However, in this example, $A_{3}$ is clearly the best choice. Therefore, the committee can recommend $A_{3}$ as the most suitable for the loading task. Furthermore, we can see that the sum of the distance of the assembly point and the other points by the PGSA is the shortest, which indicates that the points obtained by PGSA can best represent the comprehensive evaluation of experts.

Table 5 Four collective preference values

\begin{tabular}{|c|c|c|c|c|c|}
\hline Average operator & $A_{1}$ & $A_{2}$ & $A_{3}$ & $A_{4}$ & $A_{5}$ \\
\hline Ari average & {$[4.352,5.056]$} & {$[4.360,4.904]$} & {$[7.200,7.480]$} & {$[2.944,3.320]$} & {$[6.240,6.688]$} \\
\hline$\phi_{\varepsilon_{1}}$ & 4.563 & 4.523 & 7.284 & 3.057 & 6.374 \\
\hline ITWA \& DITWA & {$[4.488,5.080]$} & {$[4.728,5.392]$} & {$[7.280,7.480]$} & {$[2.992,3.360]$} & {$[6.264,6.704]$} \\
\hline$\phi_{\varepsilon_{2}}$ & 4.666 & 4.927 & 7.290 & 3.1024 & 6.396 \\
\hline ITWG \& ITWG & {$[4.400,4.968]$} & {$[4.640,5.312]$} & {$[7.168,7.448]$} & {$[2.792,3.160]$} & {$[6.184,6.648]$} \\
\hline$\phi_{\varepsilon_{3}}$ & 4.570 & 4.842 & 7.252 & 2.9031 & 6.323 \\
\hline$\phi_{\varepsilon_{4}}$ & 4.780 & 4.776 & 7.294 & 3.068 & 6.058 \\
\hline
\end{tabular}


Table 6 Sum of the distance of the assembly point and the other points

\begin{tabular}{ccccc}
\hline Aggregation method & $A_{1}$ & $A_{2}$ & $A_{3}$ & $A_{4}$ \\
\hline Ari average & $e_{1}=1.3679$ & $e_{1}=4.4222$ & $e_{1}=1.0310$ & $e_{1}=1.6885$ \\
ITWA \& DITWA & $e_{2}=1.9190$ & $e_{2}=4.0648$ & $e_{2}=1.0290$ & $e_{2}=1.6195$ \\
ITWG \& DITWG & $e_{3}=1.3473$ & $e_{3}=4.0453$ & $e_{3}=1.0415$ & $e_{3}=2.0940$ \\
PGSA & $e_{4}=1.0010$ & $e_{4}=4.0383$ & $e_{4}=1.0272$ & $e_{4}=1.6659$ \\
Distance comparison & $e_{4}<e_{3}<$ & $e_{4}<e_{3}<$ & $e_{4}<e_{2}<$ & $e_{4}=1.9134$ \\
& $e_{1}<e_{2}$ & $e_{2}<e_{1}$ & $e_{1}<e_{3}$ & $e_{2}<$ \\
\hline
\end{tabular}

Table 7 Ranking orders of different aggregation methods

\begin{tabular}{clc}
\hline Aggregation method & Ranking & $\begin{array}{c}\text { The best } \\
\text { alternative }\end{array}$ \\
\hline Ari average & $A_{3} \succ A_{5} \succ A_{1} \succ A_{2} \succ A_{4}$ & $A_{3}$ \\
ITWA \& DITWA & $A_{3} \succ A_{5} \succ A_{2} \succ A_{1} \succ A_{4}$ & $A_{3}$ \\
ITWG \& DITWG & $A_{3} \succ A_{5} \succ A_{2} \succ A_{1} \succ A_{4}$ & $A_{3}$ \\
PGSA & $A_{3} \succ A_{5} \succ A_{2} \succ A_{1} \succ A_{4}$ & $A_{3}$ \\
\hline
\end{tabular}

\section{Comparison with other methods}

The performance of the proposed method is compared with that of the ULWBM/ULWGBM [15] and ULHA/ULOWA [14] methods, respectively.

\subsection{The first comparison}

We utilize MAGDM problems in this section to illustrate the comparison. Suppose an organization plans to implement an enterprise resource planning (ERP) system [35]. Five potential ERP systems are chosen here, that is, $A_{i}(i=1,2, \ldots, 5)$ as candidates by collecting the information of both the systems and ERP vendors. The company employs a few experts or organizations as external resources for decision making. Four attributes are elected in this project to evaluate the alternatives, namely, $G_{1}, G_{2}, G_{3}, G_{4}$. The DMs evaluate the five possible alternatives $A_{i}(i=1,2, \ldots, 5)$ by using uncertain linguistic numbers under the aforementioned four attributes (weighting vector is $\boldsymbol{w}=(0.2,0.1,0.3,0.4))$ and construct the following matrix $\boldsymbol{A}=\left(\widetilde{a}_{i j}\right)_{5 \times 4}$.

We utilize the ULWBM (or ULWGBM) operator approach to address the uncertain linguistic information in MAGDM problems, and to select the most desirable candidate. Meanwhile, the proposed methods ITWA and PGSA are also used, with the comparison result shown in Table 8 . Table 9 shows that both methods provide the same best alternative, which is $A_{3}$, similar to other results.

Table 8 The overall preference values

\begin{tabular}{cccc}
\hline Alternative & ULWBM & ULWGBM & ITWA \& PGSA \\
\hline$A_{1}$ & {$\left[s_{0.71}, s_{0.98}\right]$} & {$\left[s_{1.30}, s_{1.41}\right]$} & {$\left[s_{2.40}, s_{3.36}\right]$} \\
$A_{2}$ & {$\left[s_{0.78}, s_{1.10}\right]$} & {$\left[s_{1.36}, s_{1.48}\right]$} & {$\left[s_{3.40}, s_{4.00}\right]$} \\
$A_{3}$ & {$\left[s_{1.20}, s_{1.47}\right]$} & {$\left[s_{1.52}, s_{1.60}\right]$} & {$\left[s_{4.80}, s_{5.82}\right]$} \\
$A_{4}$ & {$\left[s_{1.05}, s_{1.35}\right]$} & {$\left[s_{1.46}, s_{1.56}\right]$} & {$\left[s_{4.00}, s_{5.02}\right]$} \\
$A_{5}$ & {$\left[s_{0.81}, s_{1.12}\right]$} & {$\left[s_{1.36}, s_{1.49}\right]$} & {$\left[s_{3.36}, s_{4.80}\right]$} \\
\hline
\end{tabular}

We obtain the same sort and optimal scheme. The comprehensive value presented in Table 10 shows that the proposed method is close to the original expert evaluation value, which means that we can obtain additional information with minimal information loss.

Table 9 Ranking orders of different operator approaches

\begin{tabular}{ccc}
\hline Operator approach & Ranking & $\begin{array}{c}\text { The best } \\
\text { alternative }\end{array}$ \\
\hline ULWBM & $A_{3} \succ A_{4} \succ A_{5} \succ A_{2} \succ A_{1}$ & $A_{3}$ \\
ULWGBM & $A_{3} \succ A_{4} \succ A_{5} \succ A_{2} \succ A_{1}$ & $A_{3}$ \\
ITWA \& PGSA & $A_{3} \succ A_{4} \succ A_{5} \succ A_{2} \succ A_{1}$ & $A_{3}$ \\
\hline
\end{tabular}

Table 10 Overall preference values of the alternatives

\begin{tabular}{ccc}
\hline Alternative & ULHA \& ULOWA & ITWA \& PGSA \\
\hline$A_{1}$ & {$\left[s_{5.53}, s_{6.76}\right]$} & {$\left[s_{5.60}, s_{6.89}\right]$} \\
$A_{2}$ & {$\left[s_{5.48}, s_{7.02}\right]$} & {$\left[s_{5.65}, s_{7.00}\right]$} \\
$A_{3}$ & {$\left[s_{5.88}, s_{7.21}\right]$} & {$\left[s_{5.76}, s_{7.07}\right]$} \\
$A_{4}$ & {$\left[s_{5.34}, s_{6.55}\right]$} & {$\left[s_{5.55}, s_{6.68}\right]$} \\
$A_{5}$ & {$\left[s_{5.17}, s_{6.67}\right]$} & {$\left[s_{5.18}, s_{6.66}\right]$} \\
\hline
\end{tabular}

\subsection{The second comparison}

In this section, we illustrate the comparison by using the problem of evaluating university faculty for promotion and tenure [35]. Additionally, the attributes applied to a few universities include teaching $u_{1}$, research $u_{2}$, and service $u_{3}$. Five candidates (alternatives), $A_{j}(j=1,2, \ldots, 5)$ are evaluated by using the term set $S=\left\{s_{0}, s_{1}, s_{2}, \ldots, s_{g}\right\}$ by four DMs, $G_{k}(k=1,2,3,4)$ whose weight vector $\boldsymbol{v}=(0.24,0.26,0.23,0.27)^{\mathrm{T}}$ under the three attributes. Table 10 presents the comparison results. Table 11 shows that both methods provide the same best alternative $A_{3}$ which is similar with other results. Similar results are achieved by using the ULHA and ULOWA methods as well as the methods ITWA and PGSA proposed in this study, thereby simplifying the aggregation and making it easy to use. Therefore, the effectiveness and innovation of the methods are illustrated.

Table 11 Ranking orders

\begin{tabular}{ccc}
\hline Operator approach & Ranking & $\begin{array}{c}\text { The best } \\
\text { alternative }\end{array}$ \\
\hline ULHA \& ULOWA & $A_{3} \succ A_{2} \succ A_{1} \succ A_{4} \succ A_{5}$ & $A_{3}$ \\
ITWA \& PGSA & $A_{3} \succ A_{2} \succ A_{1} \succ A_{4} \succ A_{5}$ & $A_{3}$ \\
\hline
\end{tabular}




\section{Conclusions}

The MAGDM problem which is presented here is used to rank the alternatives or to select the most desirable one(s). An approach is proposed here to solve the MAGDM problem by using interval 2-tuple linguistic variables on the basis of the PGSA operator. The MAGDM problems are mainly reflected in two aspects. The group decisionmaking problem is based on interval 2-tuple linguistic judgment matrices. Additionally, different aggregation operators are compared in the process of preference aggregation. We provide an example for illustration and verification and compare several aggregation operators to indicate the optimality of the assembly method. In addition, we present two comparisons to demonstrate the practicality and effectiveness of the proposed method.

The contribution of this study is characterized by four aspects. First, this study proposes an intelligent optimization algorithm, which is the PGSA, to integrate uncertain linguistic information. Second, the presented method can be used not only to aggregate MAGDM problems but also to solve multi-granularity uncertain linguistic information. Third, the presented method improves the efficiency of ULWA characteristics, namely, its high reliability, easy programming, and high-speed calculation to simplify the aggregation and to make it easy to use. Finally, the proposed method combined with a few 2-tuple correlation algorithms can effectively avoid a common problem in linguistic information processing, i.e., information distortion and loss, based on its exact characteristics for linguistic information processing.

We expect to extend the proposed approach in future research by optimizing the decision-making process with more new characteristics. Potential MAGDM applications in other fields can be developed, such as medical waste management, project evaluation, and supplier selection. In addition, future decision-making theories and methods can be combined with data science, social network analysis, and other methods for cross integration between different disciplines for objective and scientific decision making.

\section{References}

[1] LIANG G S, WANG M J J. Personnel selection using fuzzy MCDM algorithm. European Journal of Operational Research, 1994, 78(1): $22-33$.

[2] XU Z S, CHEN J. Approach to group decision making based on interval-valued intuitionistic judgment matrices. Systems Engineering - Theory and Practice, 2007, 27(4): 126-133. (in Chinese)

[3] RUI Z, LI D. Fuzzy distances based FMAGDM compromise ratio method and application. Journal of Systems Engineering and Electronics, 2010, 21(3): $455-460$.

[4] LI Y, WANG Y, LIU P. Multiple attribute group decision- making methods based on trapezoidal fuzzy two-dimension linguistic power generalized aggregation operators. Soft Computing, 2016, 20(7): 2689-2704.

[5] LIU W J, LI L. Emergency decision-making combining cumulative prospect theory and group decision-making. Granular Computing, 2019, 4(1): 39-52.

[6] HERRERA F, NEZ L, SÁNCHEZ P J. Managing nonhomogeneous information in group decision making. European Journal of Operational Research, 2005, 166(1): $115-$ 132.

[7] FAN Z P, LIU Y. A method for group decision-making based on multi-granularity uncertain linguistic information. New York: Pergamon Press Ltd, 2010.

[8] HERRERA F, MARTINEZ L. A 2-tuple fuzzy linguistic representation model for computing with words. IEEE Trans. on Fuzzy Systems, 2000, 8(6): 746-752.

[9] DHOUIB D, ELLOUMI S. A new multi-criteria approach dealing with dependent and heterogeneous criteria for endof-life product strategy. Applied Mathematics \& Computation, 2011, 218(5): $1668-1681$.

[10] GONG Z W, FORREST J, YANG Y J. The optimal group consensus models for 2-tuple linguistic preference relations. Knowledge-Based Systems, 2013, 37(2): 427-437.

[11] MARTINEZ L, HERRERA F. An overview on the 2-tuple linguistic model for computing with words in decision making: extensions, applications and challenges. Information Sciences, 2012, 207(1): $1-18$.

[12] WEI G, ZHAO X. Some dependent aggregation operators with 2-tuple linguistic information and their application to multiple attribute group decision making. Expert Systems with Applications, 2012, 39(5): $5881-5886$.

[13] ZHANG H. The multiattribute group decision making method based on aggregation operators with interval-valued 2-tuple linguistic information. Mathematical and Computer Modelling, 2012, 56(1/2): $27-35$.

[14] XU Z S. Uncertain linguistic aggregation operators based approach to multiple attribute group decision making under uncertain linguistic environment. Information Sciences, 2004, 168(1): $171-184$.

[15] WEI G, ZHAO X, LIN R, et al. Uncertain linguistic Bonferroni mean operators and their application to multiple attribute decision making. Applied Mathematical Modelling, 2013, 37(7): $5277-5285$.

[16] DELGADO M, VERDEGAY J L,VILA M A. Fuzzy numbers, definitions and properties. Mathware \& Soft Computing, 1994, 1(1): $31-43$.

[17] DELGADO M, VERDEGAY J L, VILA M A. A model for linguistic partial information in decision-making problems. International Journal of Intelligent Systems, 2010, 9(4): 365-378.

[18] HERRERA F, HERRERAVIEDMA E. Aggregation operators for linguistic weighted information. New York: IEEE Press, 1997.

[19] HERRERA F, HERRERA-VIEDMA E. Linguistic decision analysis: steps for solving decision problems under linguistic information. Fuzzy Sets and Systems, 2000, 115(1): 67-82.

[20] LI L, XIE X, GUO R. Research on group decision making with interval numbers based on plant growth simulation algorithm. Kybernetes, 2014, 43(2): 250-264.

[21] LIU W, LI L. An approach to determining the integrated weights of decision makers based on interval number group decision matrices. Knowledge-Based Systems, 2015, 90: $92-$ 98.

[22] QIU J, LI L. A new approach for multiple attribute group decision making with interval-valued intuitionistic fuzzy informa- 
tion. Applied Soft Computing, 2017: S1568494617304106.

[23] XU Z S. Dependent uncertain ordered weighted aggregation operators. Information Fusion, 2008, 9(2): 310-316.

[24] LIU H C, QIN J T, MAO L X, et al. Personnel selection using interval 2-tuple linguistic VIKOR method. Human Factors and Ergonomics in Manufacturing \& Service Industries, 2015, 25(3): $370-384$.

[25] HERRERA F, MARTINEZ L. An approach for combining linguistic and numerical information based on the 2-tuple fuzzy linguistic representation model in decision-making. International Journal of Uncertainty, Fuzziness and KnowledgeBased Systems, 2000, 8(5): 539-562.

[26] LIU H C, REN M L, WU J, et al. An interval 2-tuple linguistic MCDM method for robot evaluation and selection. International Journal of Production Research, 2014, 52(10): $2867-$ 2880.

[27] ZHANG H M. Some interval-valued 2-tuple linguistic aggregation operators and application in multiattribute group decision making. Applied Mathematical Modelling, 2013, 37(6): $4269-4282$.

[28] BICUDO P, CARDOSO M. Iterative method to compute the Fermat points and Fermat distances of multiquarks. Physics Letters B, 2009, 674(2): 98-102.

[29] MORDUKHOVICH B, NAM N M. Applications of variational analysis to a generalized Fermat-Torricelli problem. Journal of Optimization Theory \& Applications, 2011, 148(3): $431-454$.

[30] TONG L, WANG C F, WANG W B, et al. A global optimization bionics algorithm for solving integer programming plant growth simulation algorithm. System Engineering Theory and Practice, 2005, 25(1): 76-85. (in Chinese)

[31] TONG L I, WANG Z T. Application of plant growth simulation algorithm on solving facility location problem. Systems Engineering - Theory and Practice, 2008, 28(12): 107-115. (in Chinese)

[32] DURMUS A, GUNEY K, BASBUG S. A plant growth simulation algorithm for pattern nulling of linear antenna arrays by amplitude control. Progress In Electromagnetics Research B, 2009, 17(17): 69-84.

[33] WANG C, CHENG H. Transmission network optimal plan- ning based on plant growth simulation algorithm. International Transactions on Electrical Energy Systems, 2010, 19(2): $291-$ 301.

[34] RAO R S, NARASIMHAM S V L, RAMALINGARAJU M. Optimal capacitor placement in a radial distribution system using plant growth simulation algorithm. International Journal of Electrical Power and Energy Systems, 2011, 33(5): 1133 1139.

[35] LIAO X, LI Y, LU B. A model for selecting an ERP system based on linguistic information processing. Information Systems, 2007, 32(7): $1005-1017$.

\section{Biographies}

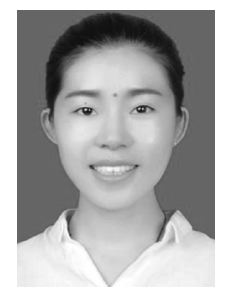

ZONG Mengting was born in 1991. She is currently a Ph.D. candidate at the Department of Business School, Nanjing University. Her research interests include group decision making, social network analysis, social computing experiments and digital innovation.

E-mail: zongmengting@163.com

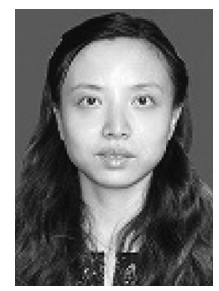

SHEN Tian was born in 1978. She is a Ph.D. and an associate professor in Nanjing University of Traditional Chinese Medicine. Her research interests include medical services management, and simulation systems.

E-mail: shentian025@aliyun.com

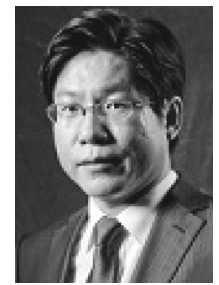

CHEN Xi was born in 1977. He is a Ph.D. and a professor in Nanjing University. His research interests include information management, big data analysis and electronic business.

E-mail: doctor_chan@163.com 